concerning pressure reduction of intussusception. ${ }^{1}$ In this paper the success rate for reduction was quoted at $76 \%$ when barium was used and $64 \%$ when air was used, giving an overall success rate of $66 \%$.

I would like to point out that the cases reported by den Hollander and Burge spanned the time that the Wessex Department of Paediatric Radiology was converting from the use of barium to air as a reduction medium, and we were relatively inexperienced in the use of air. With increasing experience our success rate has improved considerably, and a recent audit of 58 intussusception reductions performed in our department showed that the reduction rate with air had increased to $86 \%$. This compares well with figures published from all other centres as far as I am aware.

J FAIRHURST

Wessex Department of Paediatric Radiology, Southampton General Hospital,

Tremona Road,

Southampton SO9 $4 X Y$

1 Den Hollander D, Burge DM. Exclusion criteria and outcome in pressure reduction of intussusception. Arch Dis Child 1993; 68: 79-81.

\section{A career in paediatrics? A survey of paediatric senior house officers in England and Wales}

EDITOR,-Barker and Buss documented that $32 \%$ of senior house officers (SHOs) in their study reported below average levels of in-post training. ${ }^{1}$

The Department of Health and the Northern and East Anglian Postgraduate Deans have funded a pilot study in Cambridge, Norwich, Newcastle, and Sunderland to improve paediatric training for SHOs. The strength of the scheme is that it is integrated with the SHO's working day. The scheme which started on 1 March 1993 is called 'Service-Based Learning'2 and is divided into four parts. The first is 'practice based learning' and involves spending about 15 minutes discussing with the SHOs a topic of interest that arose on a ward round or an outpatient clinic. A learning plan is then devised, which may involve reading a departmental protocol or visiting a specialist clinic, or undertaking any other activity to complete the study of that topic. The second component comprises 'brief learning materials' (BLMs) and is due to start shortly. There are over 200 BLMs which cover the paediatric curriculum and which were written by 75 registrars and consultants for this study. They are symptom orientated, often have a question and answe format, and take about 15 minutes to read. The BLMs are organised in topic groups, each of which has an associated self assessment. Model answers are held by seniors. The learning materials are located on the wards and are for reading by the SHOs during gaps in the day and while on call. The third component covers departmental meetings. These must be interactive with discussion, rathe than formal 'spoon feeding' type lectures. The fourth constitutes a period of study leave of up to one week, which must be arranged at the beginning of the post.

The SHOs have a booklet in which they record what they have learnt and in which the registrar and consultant sign up the learning periods. They also meet regularly with the clinical tutor to ensure they are progressing satisfactorily. The aim is to accumulate 100 or more learning credits, each credit being equivalent to one hour's learning. Upon achieving 100 credits the SHO is awarded a service based learning certificate which should be mentioned in their curriculum vitae.

If this pilot study proves successful the scheme will be introduced to other regions. Together with the changes that will accompany the Calman report we hope that this scheme will improve the paediatric training of SHOs and make their jobs more interesting.

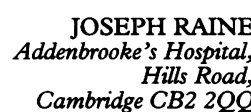
JANET GRANT
foint Centre for Education in Medicine Foint Centre for Education in Medicine,
(British Postgraduate Medical Federation/Open University) 33 Millman Street,
London WC1N $3 E \mathcal{F}$

1 Barker DP, Buss PW. A career in paediatrics? A survey of paediatric senior house officers in
England and Wales. Arch Dis Child 1993; 68: 752-3.

2 Grant J, Marsden P. Training senior house officers by service-based learning. BPMF, London: The Joint Centre for Education in Medicine, 1992.

The Australian NHMRC Twin Registry: a resource for paediatric research

EDITOR,-The Australian NHMRC Twin Registry contains over 4000 pairs of twins under 10 years old, and a further 3000 up to the age of 18 years. The parents of these twins have volunteered to consider requests from researchers for studies in bona fide projects approved by the registry. This represents a major resource for studies in paediatric and adolescent epidemiology.

There are numerous and ingenious ways in which twins can be used to address scientific and medical questions. Some examples include examining genetic and environmental causes of variation, co-twin control studies based on disease discordant or exposure discordant pairs, longitudinal studies, and studies of gene-environment interaction. ${ }^{1}$

Although there have been over 70 projects conducted over the last 12 years on the 17000 adult pairs on the registry, to date there have been few studies making use of the younger pairs. Baseline information has been computerised on about $50 \%$ of these pairs.

I would like to bring this to the attention of international paediatric researchers. Applications to utilise the registry can be made from researchers throughout the world. There may be some charge, depending on costs and the level of work required by local staff. All applications are reviewed by the executive committee of the registry, and advice on study design and practicalities is available. For more information, please write to me at the address below.

JOHN L HOPPER Australian NHMRC Twin Registry, 151 Barry Street, Carlton, Victoria 3053, Australia

1 Clifford CA, Hopper JL. The Australian NHMRC Twin Registry. A resource for the Australian scientific community. Med $\mathcal{F}$ Aust 1986; 145: 63-5. 